\title{
Germanica
}

\section{Thomas Manns Polemik mit Theodor Lessing}

La polémique entre Thomas Mann et Theodor Lessing

Thomas Manns Polemik mit Theodor Lessing

\section{Jacques Darmaun}

\section{CpenEdition}

\section{Journals}

Édition électronique

URL : http://journals.openedition.org/germanica/3788

DOI : 10.4000/germanica.3788

ISSN : 2107-0784

\section{Éditeur}

Université de Lille

\section{Édition imprimée}

Date de publication : 30 juin 2017

Pagination : 167-177

ISBN : 9782913857391

ISSN : 0984-2632

\section{Référence électronique}

Jacques Darmaun, „Thomas Manns Polemik mit Theodor Lessing“, Germanica [Online], 60 | 2017,

Online erschienen am: 30 Juni 2019, abgerufen am 07 Januar 2021. URL: http://

journals.openedition.org/germanica/3788 ; DOl: https://doi.org/10.4000/germanica.3788 


\title{
Thomas Manns Polemik mit Theodor Lessing 1
}

\author{
Jacques DARMAUN \\ Université de Nice Sophia Antipolis
}

Seit Erscheinen des Romans Buddenbrooks. Verfall einer Familie (1901) ist Thomas Mann berühmt. Anerkennung heißt für ihn vor allem exponiert zu sein. Er ist verletzbar, weil er diesen Ruhm immer neu zu erkämpfen, sich und der Welt zu beweisen hat. Von sich selbst verlangt er das Höchste, er gibt sich nicht mit Durchschnittlichem zufrieden. Dass es dabei zu Streit mit anderen Künstlern, auch mit dem eigenen Bruder, diesem anderen Ich, mit Kritikern und jüdischen Intellektuellen kommt, verwundert nicht.

Im Januar 1910 bricht eine Polemik zwischen Thomas Mann und Theodor Lessing offen aus, die schon lange schwelt. Thomas Mann hatte gerade seine Arbeit am Felix Krull unterbrochen, seine Frau war wieder schwanger, beide mit der Suche nach einer geräumigeren Villa beschäftigt. Warum lässt er sich auf diese Fehde ein? Zwei Thomas-Mann-Biografen ${ }^{2}$ schweigen dazu oder stellen diese Frage

1. - Dieser Artikel ist eine leicht veränderte Fassung von Jacques Darmauns Beitrag in dem von Elke-Vera Kotowski editierten Sammelband „Geschichte als Sinngebung des Sinnlosen". Zum Leben und Werk des Kulturkritikers Theodor Lessing (1872-1933) (Haskala, Hildesheim 2006). Germanica bedankt sich sehr herzlich bei Elke-Vera Kotowski für die freundliche Genehmigung des Nachdruckes.

2. - Klaus Harpprecht, Thomas Mann. Eine Biographie, Hamburg, Rowohlt, 1995; Donald A. Prater, Thomas Mann. Deutscher und Weltbürger. Eine Biographie, 
nicht. Lediglich Hermann Kurzke ${ }^{3}$ vermutet, Theodor Lessing müsse von Thomas Manns heimlichsten Veranlagungen gewusst haben und stichele deshalb gezielt und bewusst ganz im Stile der Zeit gegen alles Weiblich-Weibische, Unmännlich-Schwächliche an dem Schriftsteller namens Mann, der für das starke Leben nicht Manns genug sei ${ }^{4}$.

Der Literaturkritiker Samuel Lublinski hatte als einer der ersten, und zwar schon 1904, Thomas Manns Talent entdeckt, den Roman Buddenbrooks mit einer außerordentlich wohlwollenden Kritik bedacht und Thomas Mann als einen epochalen Schriftsteller der Moderne gepriesen. 1908 bescheinigt Samuel Lublinski wiederum Lob, diesmal für Thomas Manns Bühnenstück Fiorenza. Soweit die Vorbemerkungen.

Am 20. Januar 1910 wird nun Samuel Lublinski von Theodor Lessing in der Wochenschrift Die Schaubühne scharf angegriffen und diffamiert. Theodor Lessings Artikel lautet: Samuel zieht die Bilanz und Tomi melkt die Moralkuh oder zweier Könige Sturz. Eine Warnung für Deutsche, Satiren zu schreiben. Mit literarischen Beiträgen von Thomas Mann, Samuel Lublinski und den vierzig sittlichsten deutschen Dichtern und Denkern ${ }^{5}$. Es handelt sich um eine grobe, zweideutige, judenfeindlich anmutende Attacke auf Lublinski. Zahlreiche Dichter und Intellektuelle, unter ihnen Stefan Zweig, Theodor Heuss, Ernst Lissauer, verfassen umgehend ein Protestschreiben, das Thomas Mann allerdings nicht weit genug geht und das er auch nicht unterzeichnet. Erst als Theodor Lessing eine Aufforderung zum Einlenken strikt abweist, holt Thomas Mann im Literarischen Echo vom 1. März unerwartet heftig aus.

Thomas Mann gibt Theodor Lessing wieder: Samuel Lublinski sei

[...] ein verleumderisches Zerrbild, wie es roher und insipider unmöglich zu denken ist. Da wird uns eine „kleine, kugelige Gestalt“, ein „gestikulierendes“, „purzelndes“ Etwas auf „kurzen, fahrigen Beinchen“ sichtbar zu machen gesucht, ein „fettiges Synagöglein“, das sein schwammiges Bäuchlein ... wie die Apsis weit in die Außenwelt hineinstreckt, ein „Männlein“, das „,nicht sieht, nicht hört, nicht schmeckt, nicht riecht", sondern nur „,redet und schreibelt", ein „Gebürtchen“, das „sich gar naiv ins Zimmer mauschelt", mit den Beinchen „mauschelt", mit den Ärmchen „,mauschelt“, „Wortwürmlein“ nach rechts und links fallen lässt $[\ldots]^{6}$.

München/Wien, Hanser, 1995.

3. - Hermann Kurzke, Das Leben als Kunstwerk. Eine Biographie, Frankfurt a. M., Fischer, 2002.

4. - Ebd., S. $224 \mathrm{f}$.

5. - Theodor Lessing, Samuel zieht die Bilanz und Tomi melkt die Moralkuh oder Zweier Könige Sturz - Eine Warnung für Deutsche, Satiren zu schreiben, Hannover, Verlag des „Antirüpels“, 1910.

6. - Thomas Mann, Gesammelte Werke in dreizehn Bänden, Frankfurt a. M., 
Thomas Mann empört sich: Theodor Lessing sei das Gegenteil eines echten Kritikers und habe nicht einmal gelesen, wovon er rede 7 . Die Art, wie er sich seinen Glaubensbruder vornehme, spreche Bände.

Thomas Mann erbost sich über das Zerr- und Spottbild eines Juden über einen Juden, rühmt hingegen den von Theodor Lessing angegriffenen Lublinski, dessen Intelligenz, Klar- und Weitsicht. Doch das reicht noch nicht, Thomas Mann fügt weiter hinzu:

Herr Lublinski ist kein schöner Mann, er ist Jude. Aber ich kenne auch Herrn Lessing (wer kann für seine Bekanntschaften!), und ich sage nur soviel, daß, wer einen Lichtalben oder das Urbild arischer Männlichkeit in ihm zu sehen angäbe, der Schwärmerei geziehen werden müßte. [...] Wer sich als Schreckbeispiel schlechter jüdischer Rasse durchs Leben duckt, verrät mehr als Unweisheit, verrät schmutzige Selbstverachtung, wenn er sich für Pasquille bezahlen lässt, deren drittes Wort „mauscheln“ lautet. Im Stile des wildgewordenen Provinz-Feuilletons über den „espritjüdischen“ Typus zu satirisieren, steht prächtig dem zu Gesicht, der selber in aller Welt nichts weiter als das schwächste und schäbigste Exemplar dieses in einigen Fällen doch wohl bewunderungswürdigen Typus vorzustellen vermag! [...] Ich oder ein anderer: Irgendwer mußte den Schächer strafen. Kein ehrenvolles Geschäft; aber vornehmes Übersehen ist nicht immer am Platz und macht den Lumpen das Handwerk zu leicht $[\ldots]^{8}$.

Die Auseinandersetzung mit dem Schriftsteller und Philosophen Theodor Lessing erscheint also von vornherein in greller rassischer Beleuchtung und wirft zugleich die Frage nach der problematischen Situation der Juden im damaligen Deutschland auf9.

Freilich dürfen diese Sätze nicht isoliert betrachtet, keine vorschnellen Urteile gefällt werden. Dennoch wirkt Thomas Manns Entgegnung unangemessen, peinlich und weist auf Beschränktheit und Vorurteile, die unbeabsichtigt dem Antisemitismus der damaligen Zeit Vorschub leisten. Theodor Lessing wird zum „Schreckbeispiel schlechter jüdischer Rasse“ (XI, 724) etikettiert. Indem Thomas Mann Theodor Lessings antijüdische Beschimpfungen aufnimmt und auf ihn zurïckschleudert, begeht er denselben Fehler wie sein Kontrahent. Es mag wohl sein, dass Theodor Lessing, obwohl selbst Jude, sich einer antisemitischen Sprache

Fischer, 1960-1974, Band XI, S. 721 (Die Bandangabe erfolgt mit römischen, die Seitenangabe in arabischen Ziffern).

7. - Ebd., XI, S. 720.

8. - Ebd., XII, S. 723ff.

9. - Über die Entwicklung von Thomas Manns wechselnden Einstellungen zum Judentum siehe Jacques Darmaun, Thomas Mann, Deutschland und die Juden, Tübingen, Max Niemeyer Verlag, 2003. Oder Jacques Darmaun, Thomas Mann et les Juifs, Bern, Berlin, Frankfurt/M., New York, Paris, Wien, Peter Lang 1995. 
bedient, weil er sich dabei über jeden Vorwurf des Antisemitismus erhaben dünkt, und es mag sein, dass auch Thomas Mann meint, als Philosemit gegen jede Beschuldigung der Judenfeindlichkeit gefeit zu sein.

Am Antisemitismus seines Kontrahenten Theodor Lessing rügt Thomas Mann lediglich parteiische Einseitigkeit, hält sich gar für objektiv und spart nicht mit Lob über ,,anständige“ Juden wie Lublinski. Thomas Mann scheut sich auch nicht, in Theodor Lessings Fall den gegnerischen Jargon zu übernehmen. Theodor Lessing verkörpert für Thomas Mann den Inbegriff des Juden, der den judenfeindlichsten Denkschablonen entspricht.

Thomas Mann verfängt sich im Glauben an Rassen und an Völkerpsychologie in paradoxen Zweideutigkeiten. Er verteidigt die Rassenmischung. Insofern ist er kein Rassist und erst recht kein Rassenideologe, der die Überlegenheit der einen oder der anderen Rasse preist. Aber von den spezifischen Eigenheiten eines jeden ist er fest überzeugt. Eine solche Einstellung schwächt freilich jede Abwehr, macht ihn dem Antisemitismus gegenüber waffenlos und gibt dem antijüdischen Ressentiment teilweise sogar recht. Thomas Manns Selbstgerechtigkeit ist also trügerisch; er meint zwar, sowohl das Gute als auch das Schlechte ausbalanciert zu erfassen, bleibt aber dennoch im Klischee jüdischer Eigenschaften, ja einer jüdischen Natur befangen. Damit unterstellt er, die Juden seien eben definierbar, im Guten wie im Bösen.

Thomas Manns Artikel wirkt judenfeindlich, obwohl der Verfasser sich in einer Lebensphase befindet, in der ihn gerade jeglicher Antisemitismus ärgert und er sich selbst für philosemitisch hält, weil er grobe Stereotypien abgelegt zu haben meint. Judenhass bei einem Juden ist ihm unerträglich, sodass er die Selbstkontrolle verliert und ihm die Sprache des Gegners in die Feder fließt. Er übertreibt wie auch Theodor Lessing.

Die Selbstverachtung eines Juden begreift und akzeptiert Thomas Mann nicht, um so weniger als er jüdische Herkunft für eine Auszeichnung hält, allerdings eine, die verpflichtet. Aus Sicht des Schriftstellers heißt Hader mit der eigenen Abstammung Feigheit, Verrat. $\mathrm{Zu}$ seinem Deutschtum und Protestantismus bekennt sich unser Hanseat gern. Ähnliches erwartet er auch von einem Juden. Unbeholfen verübelt der Patrizier Thomas Mann dem Juden Theodor Lessing Minderwertigkeitskomplexe, ohne zu bedenken, dass eine Minderheit unter dem zweitausendjährigen Druck einer feindlichen Mehrheit verständlicherweise dazu getrieben werden kann, sich selbst zu hassen. Der Wunsch nach Assimilation entspricht einem Balanceakt zwischen Selbstverleugnung und Selbstbehauptung. Ein vermeintlicher Philosemit urteilt hier leichtfertig und unbedacht als Sohn seiner Zeit und verkennt die reale Situation der Juden völlig. Obwohl mit einer 
Frau jüdischer Herkunft verheiratet, steht Thomas Mann vor jüdischer Befindlichkeit hilflos und verständnislos da, ein Zeichen recht mangelhafter deutsch-jüdischer Symbiose in jenen Jahren.

Theodor Lessing hat nach Erscheinen von Thomas Manns Gegenschrift diesen telegraphisch zum Duell herausgefordert. Thomas Mann geht darauf nicht ein, worauf ihn Theodor Lessing als unmännlich abqualifiziert und ein ,feines, blasses Bürgerprinzchen" 10 schimpft: „So eine stille, späte Goldschnittseele, nicht vom Weibe geboren; wohl von der lieben Mama bei Wertheim in der, Abteilung für feine kunstgewerbliche Raritäten" billig und mit Geschmack alt-eingekauft [...]"11.

Der Krieg geifernder Gemeinheiten zwischen Thomas Mann und Theodor Lessing geht weiter. Wie schon erwähnt, zielen Theodor Lessings Attacken womöglich auf Thomas Manns geheimste Blößen, als würde Lessing durch Dritte über Thomas Manns sorgsam und schamhaft verborgene Veranlagungen Bescheid wissen. Es fragt sich überhaupt, ob Lessing Thomas Mann Lebensschwäche unterstellt oder provozierend auf Homoerotisches zielt, wenn er Thomas Mann der Unmännlichkeit bezichtigt. Thomas Mann sieht sich in jenen Jahren umso mehr genötigt, das Heldentum der Schwachen, eines seiner bevorzugten Motive im Frühwerk, zu betonen und die männliche Tapferkeit seiner Gestalten mit Geistesstärke und Leistungsethik hervorzuheben.

Hier liegt einer der Gründe für die Verbissenheit auf Thomas Manns Seite. Unser Autor fühlt sich da getroffen, wo er sensibel ist. Er selbst hört nicht auf, Gericht über sich zu halten und an seinem Talent zu zweifeln. Das erklärt Thomas Manns Verletzbarkeit bei Urteilen von Außenstehenden, die seinen unermüdlichen, zermürbenden Kampf zur Selbstüberwindung nicht in Betracht ziehen. Die Fragen nach Thomas Manns Lebens- und künstlerischer Tauglichkeit öffentlich zu stellen steht seinem Empfinden nach einem Theodor Lessing nicht an.

Im Zusammenhang mit anderen Widersachern zählt Thomas Mann viel später - im November 1918 auch Theodor Lessing auf: ,[...] mein geborener Feind, ein notwendiger Verächter oder doch Verächtlichmacher meiner Existenz [...]“ (Tb1, 55) ${ }^{12}$, lenkt indes sogleich wieder ein: „Ich würde mich nicht ,getroffen' fühlen, wenn nicht alle Voraussetzungen zu solcher Kritik meiner Natur in mir selber wären“ (Tb1, 55).

10. - Zitiert nach Hermann Kurzke, Thomas Mann. Das Leben als Kunstwerk, a.a.O., S. 227.

11. - Ebd., S. 227.

12. - Thomas Mann, Tagebücher 1918-1921, Frankfurt a. M., Fischer, 1979 (Tb = Tagebuch. Die arabische Ziffer gibt den Band an, die nach dem Komma folgende Ziffer die Seite). 
All das bezeugt Krampf, gekränkte Eitelkeit, verletzte Empfindlichkeiten auf beiden Seiten. In einem Brief an den Bruder Heinrich bedauert Thomas, auf den „erbärmlichen Tropf"13 eingegangen zu sein. Theodor Lessing seinerseits profitiert davon, durch den bekannten Autor in den Vordergrund geschoben zu werden, und schimpft überreizt auf Thomas Manns Egozentrismus, seine „etwas feminine, dekadente"14 Art, kratzt die Bürgerlichkeit an, die Thomas Manns existentieller Gefährdung äußeren Halt gibt. Theodor Lessing scheut sich nicht, herzlos-bissig über Thomas Manns Schwester Carla herzuziehen, diese „Chaiselongueexistenz mit heroischer Sehnsucht nach einem Millionär, mit der Politur ihrer sehr schönen Hände und vieler Romanlektüren [...]"15. Lessing bleibt nach Kräften bemüht, und er ist übrigens nicht der einzige ${ }^{16}$, Thomas Mann zur Schwiegerfamilie Pringsheim zu schlagen und ihn damit zu einem Juden abzustempeln, wogegen sich Thomas Mann immer wieder heftig zur Wehr setzt.

Auch Martin Buber u.a.m. ergreifen für Lublinski und gegen Lessing Partei, aber mit einer anderen Argumentation. Thomas Manns gehässigen Schmähungen kann Buber keineswegs beipflichten. Er gibt dem Romancier ganz und gar unrecht ${ }^{17}$.

Worin liegt der Grund für solche Hartnäckigkeit auf beiden Seiten? Reizt Theodor Lessing Thomas Manns großbürgerlicher Habitus und Lebensstil, beneidet er ihn um seine literarischen Erfolge, um die Einheirat in eine reiche Familie, mit der Lessing sich verbunden glaubt und die ihn gefördert hat? Gewiss ist Lessing ein Rebell, provoziert und spielt gerne den Bürgerschreck. Dabei vertritt der Patrizier Thomas Mann womöglich in seinen Augen den Inbegriff von Bürgerlichkeit und Selbstzufriedenheit. Für einen Spießer aber will Thomas Mann nicht gehalten werden. Überlassen wir der Lessing-Forschung die richtige Beantwortung solcher Fragestellung.

Was Thomas Manns Groll anbelangt, so geht es hier um mehr als nur um die Person Theodor Lessings. Es geht ihm um einen Menschenschlag: Thomas Mann sieht in Lessing den Querulanten, Störenfried und Schnorrer par excellence, den Parasiten in jeder Hinsicht, einen würdelosen, sich aufblähenden Feuilletonisten. Lessing vertritt gerade damals

13. - Zitiert nach: Klaus Harpprecht, Thomas Mann. Eine Biographie, a.a.O., S. 281.

14. - Ebd., S. 282.

15. - Hermann Kurzke, Thomas Mann. Das Leben als Kunstwerk, a.a.O., S. 225.

16. - Der Antisemit Adolf Bartels versucht 1910 und 1913 mit böswilliger Absicht, Thomas Mann eine jüdische Abkunft zu unterstellen, gleichermaßen der antisemitische „Semi-Kürschner oder literarisches Lexikon jüdischer Namen, Rasse und Versippung“ (1813-1913) in Deutschland (Hrsg. Stauff, Philipp, Berlin 1913).

17. - So in einem Briefentwurf, der zitiert wird in: Volkmar Hansen, Thomas Manns Heine-Rezeption, Hamburg, Hoffmann und Campe, 1975, S. 148. 
den Antipoden des in Thomas Manns Sicht erstrebenswerten guten Literaten.

Herr Lessing [...] gehört zu jenem heute weit verbreiteten Schlage von Literaten, welche Wert und Würde der Literatur nach ihrer eigenen - freilich unachtbaren - Person beurteilen und denen darum der Ehrenname des Literaten zum Schimpfwort geworden ist, das sie einander in den Journalen nachrufen und das keiner auf sich sitzen lassen will. (XI, 720)

Auf Theodor Lessing konzentriert sich der Ärger über jene Künstler und Intellektuelle, die Thomas Manns hohen Ansprüchen nicht entsprechen. Die Tatsache, dass Theodor Lessing ein Jude ist, steigert den Zorn, weil Thomas Mann in den damaligen Jahren mit guten Absichten die intellektuellen Juden zu Geistesadel verpflichtet. Lessings Kampf gegen den zersetzenden, lebenszerstörenden Geist, wie es im Untergang der Erde am Geist ${ }^{18}$ heißt, kommt Thomas Mann einem Treuebruch, einem Mangel an Redlichkeit dem Judentum gegenüber gleich. Lessing erhält somit eine Sündenbockfunktion für Thomas Manns verdrängte antijüdische Phobien. Lessing wird nichts verziehen. Der Schriftsteller unterstellt Lessing sogar eine auffällige Hässlichkeit, was dem heutigen Betrachter von damaligen Fotos nicht unbedingt einleuchten muss.

Auch Theodor Lessings Schriften gegenüber legt Thomas Mann Unverständnis an den Tag. Lessings Kassandrarufe auf den Fortschritt und die Zivilisation muten in mancher Hinsicht sehr aktuell an. Seine Zivilisationskritik wäre auch heute noch durchaus vertretbar. Trotz aller Wut auf Lessings Kulturkritik sind die Positionen beider Kontrahenten gar nicht so weit von einander entfernt. Ein Beispiel: Die Hauptidee in Goethe und Tolstoi, einem 1921 verfassten und 1925 umgearbeiteten Essay Thomas Manns, ist eine Reflexion über Natur und Geist, wie sie auch in Lessings Schriften Die verfluchte Kultur. Gedanken über den Gegensatz von Leben und Geist ${ }^{19}$ und Untergang der Erde am Geist. Europa und Asien anzutreffen ist. Es entspricht durchaus dem damaligen Zeitgeist, gegen Verstand, Aufklärung und Rationalismus zu Felde zu ziehen. Lessing spricht bildlich vom Messer des Geistes, das in dem Stamm des Weltbaums stecke ${ }^{20}$ und drin stecken bleiben müsse, wolle man nicht den Baum des Lebens bluten lassen. Auch Thomas

18. - Siehe Theodor Lessing, Untergang der Erde am Geist. Europa und Asien, Hannover, W.A. Adam Verlag, 1924, 3. Auflage, S. 145.

19. - Theodor Lessing, Die verfluchte Kultur. Gedanken über den Gegensatz von Leben und Geist, München, Beck, 1921 (Neudruck: Theodor Lessing, Die verfluchte Kultur. Gedanken über den Gegensatz von Leben und Geist, München, Matthes \& Seitz, 1981).

20. - „Geist ist eingedrungen in die Natur, wie das Messer dringt in eines Baumes Mark.“ In: Theodor Lessing, Untergang der Erde am Geist, a.a.O., S. 127. 
Mann hegt gewisse Vorbehalte gegen den Intellekt. Der Gegensatz von Natur und Geist gilt zwar immer noch, aber gerade auf Bewahrung, Vermittlung zwischen beiden Seiten kommt es Thomas Mann an. Auch Theodor Lessing sucht Maß und Ausgleich.

Als dann aber die Lebensphilosophie, die Apologie von Blut und Boden, die präfaschistoide, mythisierende, irrationale Ideologie überhandnehmen, hört Thomas Mann nicht auf, sie zu bekämpfen, indem er humanistische Positionen bezieht. Lessing muss nun unverdienterweise als Zielscheibe herhalten, als vertrete gerade er die feindliche Phraseologie und die geistige Vorläuferschaft der späteren Entmachtung der Vernunft. Thomas Mann hält Lessing für den Feind des Geistes, der er nicht ist. Theodor Lessing wie auch Thomas Mann verhöhnen übertriebenen Geistesdünkel, auch Lessing weiß sehr wohl um die Unentbehrlichkeit des Geistes für das Leben. Lessings Kulturkritik zielt aber nicht darauf ab, Kultur umzubringen, wie es die Nazis taten. Doch gerade das wirft Thomas Mann Lessing vor und stellt ihn dabei mit seinen späteren Henkern in ein und dieselbe Reihe.

Den blinden Hass auf Lessing hat Thomas Mann sein Leben lang nur schwer zu bezähmen vermocht und nicht einmal bei der Nachricht von der Ermordung Lessings zu bändigen gewusst. Am 1. September 1933 notiert der Schriftsteller feindselig in sein Tagebuch: „Mir graust es vor einem solchen Ende, nicht weil es das Ende, sondern weil es so elend ist und einem Lessing anstehen mag, aber nicht mir.“ (Tb2, 165) In einem Brief an den Sohn Klaus heißt es mitleidslos über den Ermordeten: „War immer schon ein falscher Märtyrer." ${ }^{21}$ Noch 1934 beschäftigt Lessings Tod den Autor:

Der-übrigens recht widerwärtige Lessing, der stumpfsinniger Weise ermordet wurde, hatte ein Buch gegen den Geist geschrieben - warum musste man den ermorden. Er hatte zwar allerlei [...] Taktlosigkeiten begangen und nannte sich einen Sozialisten. In der Hauptsache aber war er einer Gesinnung mit seinen Mördern. (Tb2, 474)

Hier setzt Thomas Mann Lessing wiederum mit seinen Mördern gleich.

Die Auseinandersetzung mit Theodor Lessings Gedankengut hinterlässt Spuren in Thomas Manns Werk. Lessing muss Thomas Manns Feindbild entsprochen haben, das er in seinen frühen Erzählungen als den dilettantischen Kaffeehaus- und Möchte-gern-Literaten gestaltet hat. Mit dem zweibändigen Werk Philosophie als Tat gesellt sich Theodor Lessing zu jenen Aktivisten, die wie Kurt Hiller ${ }^{22}$ von Thomas Mann

21. - Hermann Kurzke, Thomas Mann. Das Leben als Kunstwerk, a.a.O., S. 228.

22. - Zur Polemik zwischen Thomas Mann und Kurt Hiller vgl. Jacques Darmaun, Thomas Mann, Deutschland und die Juden, a.a.O., S. 96ff. 
in den Betrachtungen eines Unpolitischen als Zivilisationsliteraten verhöhnt werden, da sie durch das Wort die Welt ändern wollen. Zu dieser Menschenspezies zählt der Verfasser der Betrachtungen eines Unpolitischen auch künstlerische und intellektuelle Modenarren, Snobs, wie Thomas Mann sie im Münchner Schwabing getroffen hat. Sie nähren seine Antipathie jenen gegenüber, die das Gebiet der Literatur verlassen, um sich in die Politik, in die revolutionären Nachkriegswirren einzumischen oder die Bayrische Räterepublik auszurufen.

Thomas Mann stemmt sich gegen ein Heidentum lebensphilosophischer Provenienz, das ihm einer Verhunzung Friedrich Nietzsches gleichkommt. Bei Juden erträgt der Schriftsteller Deutschtümelei und Nietzsche-Epigonentum am wenigsten. Friedrich Nietzsche habe keineswegs vorgehabt, den Feinden des Geistes und den Apologeten des Leibes Vorschub zu leisten. Thomas Mann betont: „Nietzsche hat zwar den Leib auf Kosten der ,Seele' verherrlicht, - aber keineswegs auf Kosten des [,]Geistes['] [...]“23.

Die Reibungen an Lessingschem Ideengut lassen sich weiter verfolgen. Die jüdische Romanfigur Naphta im Zauberberg attackiert in den Streitgesprächen mit seinem Gegenspieler Settembrini bissig die Nützlichkeitsprinzipien, greift das Abendland und den Kapitalismus an. Die Naphta-Gestalt vertritt im Roman den Inbegriff des Geistes. Ist Naphtas Vater noch der Schochet, der religiöse Schlächter, so ist der Sohn der "Schlächter“ des Geistes und verkörpert das geistige Prinzip in seiner selbstzerstörerischen Konsequenz. Er begeht schließlich Selbstmord. Die Parallele zu folgender Passage bei Theodor Lessing liegt auf der Hand:

Buddhas Willensziel: „Geist ist der Schlächter am Leben“. Wollen wir nicht den Schlächter schlachten? Ja, sich selber schlachten wird [...] der Schlächter. Sein Wille ist zuletzt der Wille, nicht zu wollen. Bewusstsein kann nur aufgehoben werden durch Bewusstsein. Das Ziel des Geistes ist - sein Erlöschen ${ }^{24}$.

Ein Bild des Fanatismus bietet auch jener ,ehemalige Bildhauer“ (III, 876), der das Projekt, „Altzeitungspapier“ (III, 877) neu zu verwerten, wie einen „Heilsgedanken“ (III, 878) verfolgt. Das Gesicht des Staatsanwalts Paravant, der die „Quadratur des Kreises“ (III, 875) lösen will, trägt „den visionären und verbissenen Ausdruck der Manie.“ (III, 875) Alle diese Figuren aus dem Zauberberg gehören in Thomas

23. - Thomas Manns Notizen zitiert Hans Wysling, ,,,Geist und Kunst“. Thomas Manns Notizen zu einem Literatur-Essay. Ediert und kommentiert von Hans Wysling“, in: Paul Scherrer, Hans Wysling, Quellenkritische Studien zum Werk Thomas Manns, Bern, München, Francke 1967, S. 169, Notiz 35.

24. - Theodor Lessing, Untergang der Erde am Geist, a.a.O., S. 144. 
Manns Augen zum Typ des Literaten im negativen Sinne des Wortes, zu den Zerstörern der Vernunft, wie man nach Lukács sagen möchte. Diese Querulanten führen mitunter Theodor Lessings Sprache, im Joseph-Zyklus vor allem die Teufelsfigur des Semael und der Teufel im Roman Doktor Faustus. In den Notizen zu der geplanten, nie erschienenen Erzählung Der Elende ist von Theodor Lessing die Rede ${ }^{25}$, der in die Titelgestalt mit eingehen sollte.

In Thomas Manns Gestalten überlappen sich oft Züge, Kennzeichen oder Merkmale mehrerer Modelle, Vorbilder aus seiner unmittelbaren Lebenswelt. Den Ärger mit Lessing überträgt der Romancier daher immer wieder auf Figuren, die mit dem Bösen im Bunde sind. Dies erscheint umso ungerechter, als die Lessing-Rezeption dem Romancier manche Anregung vermittelt haben mag. Mitunter handelt es sich um winzige Details, die in Thomas Manns Zeichensprache aufgeladen werden und motivisch wiederkehren. Ein Beispiel: In Theodor Lessings Schrift Die verfluchte Kultur fällt das Bild von der verunstalteten Natur auf, die zu einem ,wohlbestellte[n] Schachbrett der Kultur"26 verkomme. Statt Natur blieben nur „Aecker, Wiese, Felder, Wald, bewirtschaftet von Bildungsmenschen und dem, was ihnen nützlich ist [...]"27. In Thomas Manns Werk tragen mehrere Literaten schachbrettartig karierte Hosen als Andeutung darauf, dass diese Gestalten auch Hanswurst-Züge in einer Welt annehmen können, deren geistiger Horizont immer beschränkter und Fortschritt zum Rückschritt wird. Der Zivilisationsliterat und Aufklärer Settembrini aus dem Zauberberg erscheint ständig in „,hellgelblich karierten Hosen“(III, 82). Ein weiterer typischer Literat, der Übersetzer Rüdiger Schildknapp aus dem Roman Doktor Faustus, hat ,tagein, tagaus dieselben schon recht mitgenommenen gewürfelten Breesches“ (VI, 225) an. Der kranke Leverkühn, dieser deutsche Faustus, trägt in seiner geistigen Umnachtung weite, ungebügelte, klein gewürfelte Beinkleider (VI, 642), auch das ein Hinweis auf Deutschlands Niedergang von der Kultur in die Syphilisation und Zivilisation. Thomas Manns literarische Darstellung vom Untergang des Abendlandes speist sich also auch aus Lessings expressiver Sprache.

Thomas Manns Wut auf Lessing erreicht Ausmaße, die jenseits des Anstands und der Vernunft liegen. Die Anlässe und Gründe für solche Entgleisung im Affekt kennen wir. Hier offenbart sich eine Schwäche des damaligen Zeitgeistes, das weit verbreitete, pseudowissenschaft-

25. - Siehe dazu Peter de Mendelssohn, Der Zauberer. Das Leben des deutschen Schriftstellers Thomas Mann, 1. Teil, 1875-1918, Frankfurt a. M., Fischer, 1975, S. 824-832.

26. - Theodor Lessing, Die verfluchte Kultur, a.a.O., S. 9 (Neudruck: S. 18).

27. - Ebd., S. 9 (Neudruck S. 18). 
lich unterstützte Beharren auf Völkerpsychologie und Rassen, das Festhalten an vermeintlich unveränderlichen Merkmalen bestimmter Menschengruppen. Trotz der philosemitischen Absichten Thomas Manns kommt hier ein Denken zum Vorschein, das auf Verdrängtes und auf eine uralte kulturelle Erblast verweist. In Theodor Lessing sieht Thomas Mann einen Juden, der den gängigen judenfeindlichen Schablonen entspricht. Der Graben zwischen beiden Widersachern ist auch ideell nicht so tief, wie Thomas Mann es glaubt. Der hasserfüllte Gegensatz ist ein Paradox, ein fatales Missverständnis und symptomatisch für die Erschwernisse, die den einstigen deutsch-jüdischen Dialog selbst bei den besten Absichten belastet haben. 
